\title{
ISPRS \\ Detecting Building Alignments for Generalisation Purposes
}

\author{
Sidonie Christophe and Anne Ruas
}

Cogit Laboratory - IGN-France, 2 avenue Pasteur 94160 Saint Mandé France, anne.ruas@ign.fr

\begin{abstract}
Generalisation is well recognised as a complex process that should trigger specific algorithms, on different types of objects in some logical or appropriate order. To guide the process (where and how to generalise) one solution is to distinguish characterisation from the generalisation process. Characterisation aims at finding and describing relevant 'working areas' that can be a part of an object or a set of objects. As a result, the choice of an appropriate algorithm(s) becomes easier and can be constrained by the detected properties of this new entity. This paper presents a method to both detect and characterise building alignments in an effort to improve the use of generalisation algorithms namely typification and displacement. The first step consists of the identification of building alignments from straight-line templates. The second step characterises these alignments to retain only those that are perceptually regular. The characterisation is based on an analysis of the spatial location of buildings as well as on the properties of the buildings that belong to the alignment in question. To evaluate the regularity of the distribution, estimators are proposed for each property.. At the end a global quality estimator of the perceptual alignment- based on the aggregation of the estimators - is proposed. This global estimator is used to retain the best building alignments that will then be carefully generalised. The results presented have been implemented in the Lamps2 GIS software.
\end{abstract}

Keywords: pattern recognition, spatial Analysis, typification, generalisation 


\section{Introduction}

\subsection{Why Detect Building Patterns?}

There are a number of approaches for automation of the generalisation process. For example one approach followed by the GIUZ laboratory of Zurich uses constrained algorithms in which internal conditions transform information while at the same time preserve some properties. Another approach which separates the task of identification/characterisation from the task of transformation has been investigated at the COGIT laboratory for a number of years. Detecting building patterns is a well recognised characterisation issue. The intent of this paper is to report on some of the findings related to the detection and characterisation of selected groups of buildings, , and then present some specific algorithms that effectively generalise these building groups. -

The generalisation of buildings concerns both the generalisation of each building - shape simplification, size dilation and local enlargement - and involves the contextual generalisation of a set of buildings - selection, typification, aggregation, displacement. Contextual generalisation usually occurs at a medium scale, whenever the main information to preserve no longer relates to each building but rather, illustrates that some buildings exist. The challenge is to preserve the appropriate building characteristics while at the same time reduce the quantity. The detection of building patterns involves finding a relevant group of buildings that have some particular spatial location of interest and then in identifying the properties that should be preserved during the process. The operation of 'reducing the number of buildings while preserving the properties of the group, is referred to as typification.

\subsection{What Makes a Pattern?}

When viewing a map, most people are able to conceptually group entities together. The action of grouping is usually based on some similarity in the criteria. Things that 'look the same' can be grouped together. This definition of perceptual groups comes from the Gestalt theory [Wertheimer 12]. A perceptible group consists of entities that have similar characteristics and do not exhibit too many differences. The separation between two groups generally occurs whenever there are sufficient inconsistencies in pattern regularity. For example, some are considered 'strong' group members when they have an important regularity of some criteria, or are considered not strong when irregularities or inconsistencies occur. Others can be considered as 'light' either because there are some important irregularities or because the regularities are not 'excellent'. All these qualitative and fuzzy definitions are intuitive to humans but are complex for computing purposes. Moreover, working on a classification rapidly illustrates that there is no universal partitioning of entities in the mathematical sense or in any consistent computational process. The grouping process is fuzzy in its definition and fuzzy in its borders. 
The aim of this research has been to detect a particular case of building groups that can be referred to as building perceptual alignments. The geometry of the buildings used for testing is $2 \mathrm{D}$ polygons, with 1 meter of geometric resolution. A building pattern is a set of close buildings that have a 'well organised' spatial distribution and that have some geometric similarities (shape, size, and orientation). Consequently not all groups of buildings are appropriate for testing; preferable are those that are spatially organised and share perceptual similarities. Amongst the possible spatial distributions, this study focuses on alignment (aligned along a straight line) as follows;

1. It is a frequent pattern

2. It can be easily perceived and it structures the space

3. It needs to be explicitly preserved during generalisation.

This latter point is relevant since the number of operations tend to remove contextual regularity (such as displacement and object removal). Other objects such as roads, have not been integrated, as the detection of patterns was to be based on a single object category. Further, the approach was to study the relationship of buildings with roads after the identification of perceptual alignments. Roads were then subsequently considered as an object resource with which to reinforce the process. Thus, in summary, the proposed mechanism is to look for buildings that are close to one another and that are aligned, in a regular manner i.e. their distance is regular or consistent along a line. Additionally they should exhibit similarities in terms of shape, size and orientation.

A group of aligned buildings (i.e. building alignment) is therefore considered a prerequisite condition but is not all encompassing vis-à-vis 'building perceptual alignment'. The term 'perceptual' implies the regularity of the alignment. According to these definitions, however, it is clear that a perfect perceptual alignment does not exist. As a result, it is necessary to allow some flexibility in the definition of regularity and to try to quantify it as much as possible. The difficulty of such an exercise involves aspects about resolving the thresholds (i.e. what is the limit between nearly regular and not regular) and determining the aggregation of independent criteria (e.g. size and shape) that characterise the global 'quality' of the building perceptual alignment.

\subsection{Previous Work in the Generalisation Field}

Amongst previous related research, work has been undertaken in both the area of generalisation and pattern recognition. In these areas, works that compliment this particular research endeavour can be categorised into two sets and are as follows

- Research on detection of building structures for building typification;

- [Hangouët 98] detects buildings aligned along roads,

- [Regnauld 98] detects close buildings organised along a graph using Minimum Spanning tree (MST), 
- [Boffet and Rocca-Serra 01] detect and aggregate triplets of buildings to constitute building alignment using a bottom-up approach,

- Research on building typification;

- [Sester 00] randomly removes some buildings and relocates the remaining ones as regularly as possible by means of attraction forces computed in an iterative way using neural networks,

- [Ruas 99] groups buildings by means of a network (Delaunay triangulation) and progressively removes the 'worst building' by means of a cost function based on size, density and directional proximity.

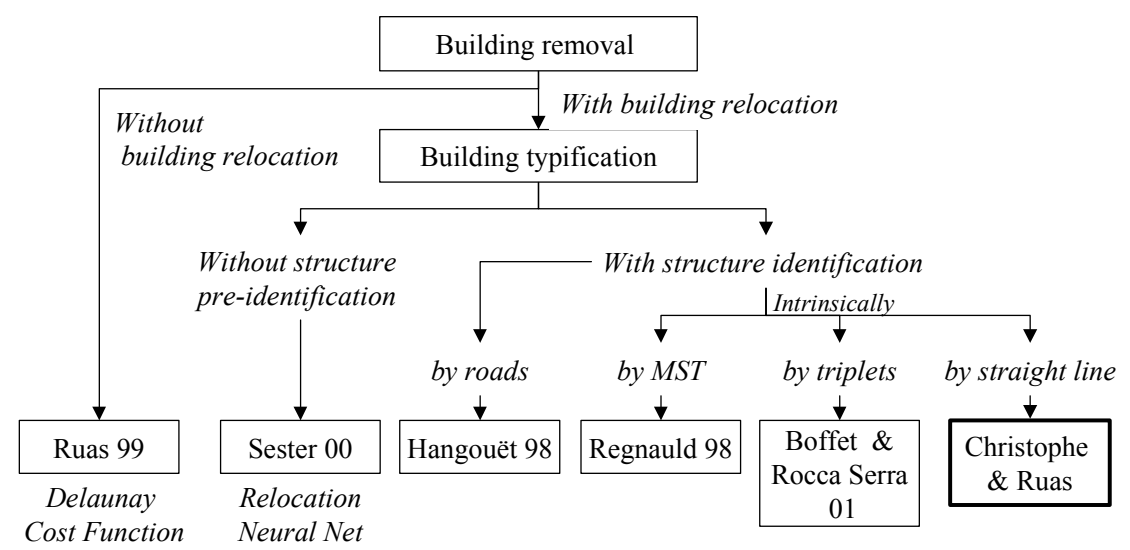

Fig. 1. Some existing methods for building removal

The method proposed here builds on a selection of work, see [Regnauld 98] and [Boffet and Rocca-Serra 01].. The challenge is to propose a method less complex than [Regnauld 98] (i.e. without requiring the computation of MST) but more robust than [Boffet and Rocca-Serra 01].

\subsection{How to Detect Building Perceptual Alignments?}

To detect a straight line alignment pattern, one can either group objects by means of a spatial structure such as MST, Delaunay or Voronoï, and determine if straight alignments exist within these linked objects, or one can use the straight line to directly group the objects. This latter method has been chosen as it provides hypothetical straight line alignments without requiring the development of complex spatial structure analysis tools.

The proposed method is divided into two steps:

1. The first step (section 2) aims at identifying aligned buildings within an urban block (a close cycle of streets). The result is a set of aligned buildings that are potential candidates for becoming building perceptual alignment. At this stage, the distance between buildings may or may not be regular. 
2. The second step (section 3) qualifies the potential candidates according to their various regularities; eliminates the ones that are clearly not regular and, quantifies the quality of the retained candidates.

Section 2 and 3 below address each of these steps. The implementation has been achieved using the GIS Lamps2 of Laser Scan.

\section{The Detection of Straight Line Building Alignment}

This section presents the methodology used and identifies straight line alignments of buildings. A top-down method has been developed. Straight lines are predefined patterns used to detect the alignment. Starting from a geometric description of alignment, we determine which buildings are concerned by their structure (2.1). Further, the structures identified are assessed and the incomplete ones are recomposed (2.2). The intent is to use an exhaustive search of all alignment possibilities by means of a one-line template that takes 'all' orientations from a single anchor point, located on the xmin and ymin of the urban block.

\subsection{The Detection of Potential Alignments Based on Projection}

It is worth noting that the orthogonal projection of aligned points gives close projected-points on a straight line of projection (Fig. 2). As such, the centroid of all buildings of the urban block is projected on an 'exhaustive' set of straight lines. Then on each line, groups of very close projected-points are searched. These groups of points represent the potential alignments. The relation between a projected point and its building is maintained throughout the process: thus, a projected point is linked to one single building.

Questions raised by this choice are:

- Which straight lines are to be used for this projection?

- How should the distribution of projected-points be assessed?

- How should the point clusters be filtered to detect the aligned buildings?

\subsubsection{Straight Lines Choice}

To find all the potential candidates, each urban block is assessed using a set of straight lines defined by an angle $\alpha$ and an anchor-point. The angle $\alpha$ varies from 0 to 180 degrees with a 1 degree step. The complete scan highlights all alignments. 


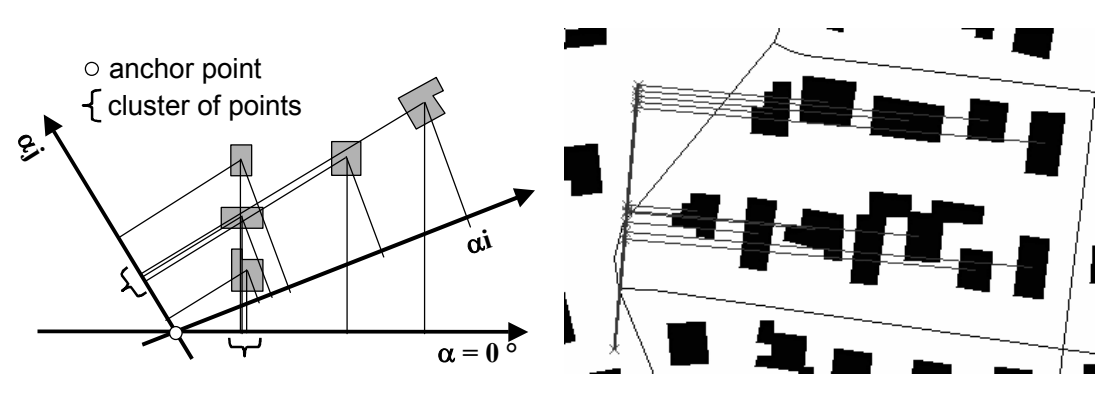

Fig. 2. Straight line assessment of an urban area

\subsubsection{Study of Distribution of Projected-Points on a Straight Line $\alpha$}

Each straight line holds a set of projected points amongst which some clusters may exist. To detect the clusters a simple method has been chosen that consists of gathering the projected-points together in equal size packs from the anchor-point and assessing the proximity within each pack. The greatest distance between two successive points in each pack - called $\delta$ max - represents the proximity inside the pack. Other methods could also have been used for this step such as an ascending hierarchical classification method.

The size of the packs refers to the number of successive points per pack. An alignment should likely be composed of at least 4 buildings. As alignments often involve a limited number of buildings, we have experimentally chosen to study structures of 4,5 and 6 points. Consequently, for a single straight line, three collections of packs are generated corresponding to size 4, 5 and 6 .

\subsubsection{Selection of the Best Packs}

At this stage, there are $180 * 3$ collections of packs to assess (line ${ }_{\alpha}=\left(\right.$ coll pack $_{\mathrm{i}}$, $\left.\delta \max _{\mathrm{i}}\right\}, \operatorname{coll5}\left\{\right.$ pack $\left._{\mathrm{j}}, \delta \max _{\mathrm{j}}\right\}$, coll6 $\left\{\right.$ pack $\left.\left.\left._{\mathrm{k}}, \delta \max _{\mathrm{k}}\right\}\right)\right)$. Most of these do not present any alignment. To filter the best lines, the minimum value of $\delta \max -\delta$ max min per collection and per line is computed. This value represents the "efficiency" of the line to identify the clusters. It is used to order the line by efficiency. Experimentally, it is apparent that all lines and collections that have a Smax min bigger than 3 meters can be rejected.

The same alignment, however, can still occur in different packs:

- An alignment of 6 buildings will be described for the same $\alpha, 3$ times in a collection of 4 points, 2 times in the collection of 5 points and 1 time in the collection of 6 points

- An alignment may be described with successive $\alpha$ values. 
Based on point's identifier, a first filter is done for each line to retain only the longest pack representing the same points. A second filtering - based on the buildings identifier- is done between lines to reject redundant alignments. We will see later on that the alignments are not perfect and at this stage are considered only a 'potential alignment'. To view alignments, a set of segments between the centroid of the consecutive buildings is created (Fig. 3, left). To find a better geometry of the straight-line alignment, the adjustment of the alignment is measured using a classical regression between centroids. A set of alignments of different sizes is obtained (Fig. 3, right).
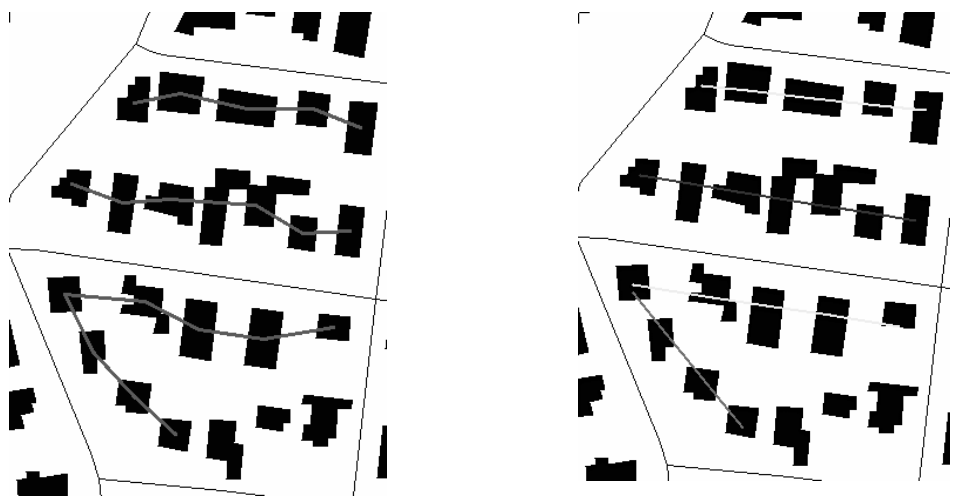

Fig. 3. Adjustment of alignments with regression (black :6, white:5, gray:4)

At the end of this step some overlapping or incompleteness still exists and should be corrected.

\subsection{The Filtering and Composition of Alignments}

The first step determined that a number of potential alignments are incomplete. As such the potential alignments and filter overlapping must be extended.

Only small alignments (maximum 6 buildings) are detected but larger ones have to be detected in their entire size (case 1). Alignments are extended to catch buildings using an iterative buffer at the extremity (of 10m) (Fig. 4). Moreover some buildings fall 'under' alignment, but are not actually a component of it (case 2). This can result either from the packs filtering or from a certain deviation of the centroid. Those buildings are caught by a buffer of $10 \mathrm{~m}$ on both sides of the alignment (Fig. 4).

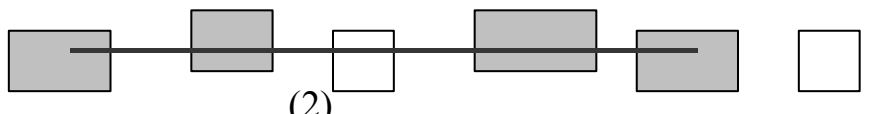

Fig. 4. Situation of alignments: recuperation and extension 
After the extension of alignments some alignments could not be merged (Fig. 5): if they have the same general orientation, they are merged and the adjustment of the alignment geometry is re-computed.

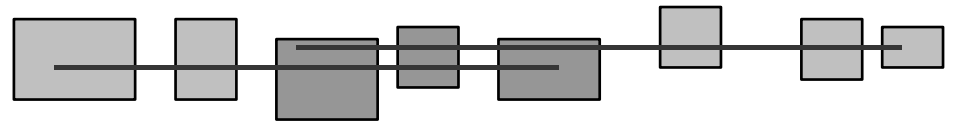

Fig. 5. Situation of alignments: merging

All the identified alignments are added to the database. The "alignment" class is created with two particular attributes: the slope of the regression and the list of component-buildings. The association between one object 'alignment' and the object 'buildings' is a composition relationship (cardinality $1: n$ ).
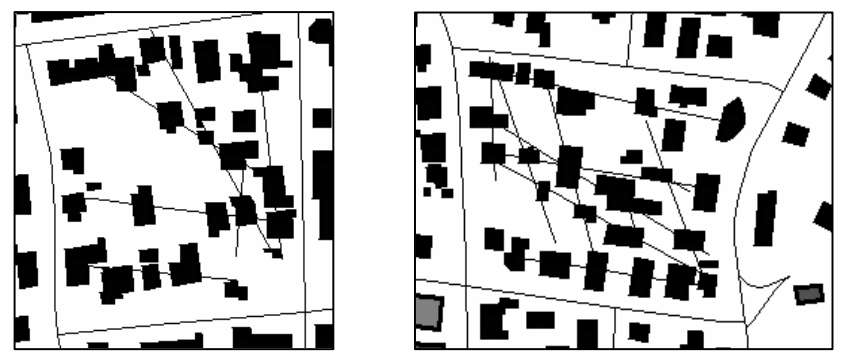

Fig. 6. Examples of results on two different urban blocks (in $0-90^{\circ}$ only)

In Fig. 6 it's apparent that an alignment is not always a perceptual structure since the inter-distances are large and irregular and/or because the buildings that belong to the alignments are different one from another. The alignments now have to be characterised to become building perceptual alignments.

\section{From Alignments to Perceptual Alignments}

The detected alignments must be qualified and ranked according to their importance. Some alignments will be rejected while others will be preserved and qualified for the typification process. Some visual criteria, based on principles of perception, are defined to describe potential alignments (3.1). The selection of the "best" alignments is based on regularity of all perceptual criteria. Results and required improvements are proposed in section 4 . 


\subsection{The Criteria of Characterisation}

Perceptual criteria are used to select and to describe the alignments. Two kinds of criteria were selected:

- The position of objects (which represent the quality of the pattern); in other words, the proximity and the alignment of their sides and centre.

- The similarity between objects (which represent the quality of the component of the pattern) and refers to the shape, the size and the orientation of buildings.

At this stage measures are not aggregated, they are simply listed.

\subsubsection{The Position of Objects}

Proximity: The proximity which is the projection (minimal distance) between the boundary of two consecutive buildings is computed (Fig. 7, left).

Arrangement of objects: Qualities of the sides of alignment and alignment of centres are computed by using angle variation.

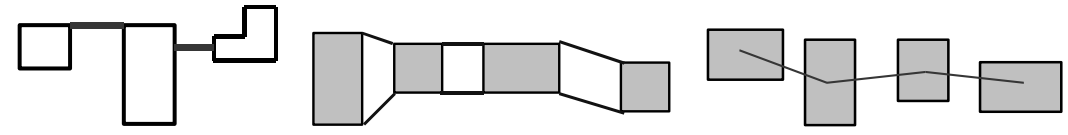

Fig. 7. Measures of proximity,alignment of sides, and, alignment of centres

\subsubsection{The Similarity of Objects}

Size: The size is the area of a building.

Shape: Refers to the concavity of buildings, using the comparison of the area and the area of the convex hull (concavity $=1$ - area/area (convex-hull)). Other measures could be added such as elongation ratio.

Orientation: Various definitions have been described in [Regnauld 98] such as the general orientation and the orientation of walls. The general orientation is measured by the average between the two longest segments between the points of the border (Fig. 8 left). This method gives approximate results as follows: first for squared buildings, the mean is proves insignificant second, for very irregular buildings, the results are invalid.

As a result, wall orientation, which is the mean of the orientation of the wall in [0, pi/2], weighted by the length of each wall has been chosen. Another orientation of interest, is the perpendicular orientation (Fig. 8 right ). 

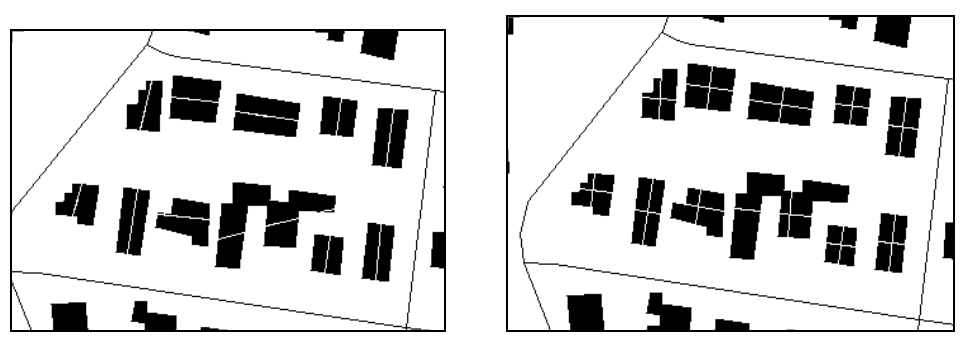

Fig. 8. Measure of general orientation and orientation of walls

The orientation of walls supports a more effective comparison of buildings and is more suitable than a general orientation.

\subsection{Aggregation of Criteria Necessary to Define Pattern Quality}

The detected alignments must now be qualified according to perceptual criteria. Each pattern is characterised by a distribution of values concerning proximity, arrangements, size, shape and orientation of the buildings. Each criterion is perceptually 'good' if the values are close one to another. The perception is thus based on the regularity of values.

An estimator of homogeneity needs to be developed for each criterion and then aggregated to a global one, for each significant pattern. Assuming that a scale of comparable values is required, a normalisation must be done. The classical normalisation of values prior to computing the standard deviation $\left(\mathrm{X}_{\mathrm{i}}=\right.$ $\left.\left(\max \left(\mathrm{x}_{\mathrm{j}}\right)-\mathrm{x}_{\mathrm{i}}\right) / \max \left(\mathrm{x}_{\mathrm{j}}\right)\right)$ did not yield appropriate results.

\subsubsection{Estimator of Homogeneity for Each Criterion}

The measure of regularity is based on the standard deviation. The mean of the values has not been used since there are only a few objects and thus, the influence of any exceptions would be too high. Consequently, in the standard deviation, the mean has been substituted with the median, which in this case, is more appropriate. Several estimators E, were then developed, one per criterion, $\sigma^{\prime}=\sqrt{ }$ $\left(1 / n * \Sigma_{i}\left(x_{i}-m e d\right)^{2}\right)$ :

When the value of $x_{i}$ is larger than 1 (area, distance), we divide the standard deviation by the median. The estimator values, however, should be comparable. If 0.5 is perceived as medium for one property, it should be perceived as medium for the others. Consequently, each estimator has to be adapted to reflect the homogeneity of each criterion:

- Arrangement, orientation and shape, $E=\sigma^{\prime}$

- Proximity, $E_{p}=\sigma^{\prime} /$ median

- Area, $E_{a}=\sigma^{\prime \prime} /$ median, with $\sigma^{\prime \prime}=\sqrt{ }\left(1 / n * \Sigma_{i}\left(x_{i}-\right.\right.$ med $\left.)\right)$. The quadratic value placed too much emphasis on large areas. 
The standard deviation only computes the regularity of the distance and not the distance itself. Consequently, if the median for proximity is superior to $10 \mathrm{~m}$, some values are added to the test value to penalise it ( $E_{p}$ can be higher than 1).

We have a set of estimators $E_{i}$ defining each criterion of the structure. The quantitative values given by each estimator are experimentally tested and validated. To clarify the results quantitative values are transformed into qualitative values. If the values of $E_{i}$ are superior to 1 it is "very bad", between 1 and 0,7 it is "bad", between 0,7 and 0,5 it is "medium", between 0,5 and 0,25 it is "good", under 0,25 it is "very good". (see Fig. 9)

Finally, a global estimator GE is proposed that defines the global quality of the pattern, measured by $1 / n * \Sigma_{i} E_{i}{ }^{2}$. The use of the square enforces the influence of small values (i.e. regular) and high values; a value $>1$, i.e. not regular at all will correspond to what is required. Experimentally, we saw that if $\mathrm{GE}<0.25$, the alignment of buildings was perceptually good. Consequently, among the alignments defined in section 2, only those having a GE value under this threshold are considered as being a 'building perceptual alignment'. Therefore, $0,25=0,5^{2}$, corresponds to the limit between good and medium values.

\subsubsection{Accounting for the Number of Buildings}

These preliminary experiments were undertaken with samples of between 4 and 15 buildings. The more elongated the pattern, the less regular it might be. The number of objects could be included in the final cost function but this requires further assessment.

\subsection{Results and Comments}

A methodology has been presented to detect and qualify building perceptual alignments. Some comments on the limitations and necessary improvements are briefly outlined.

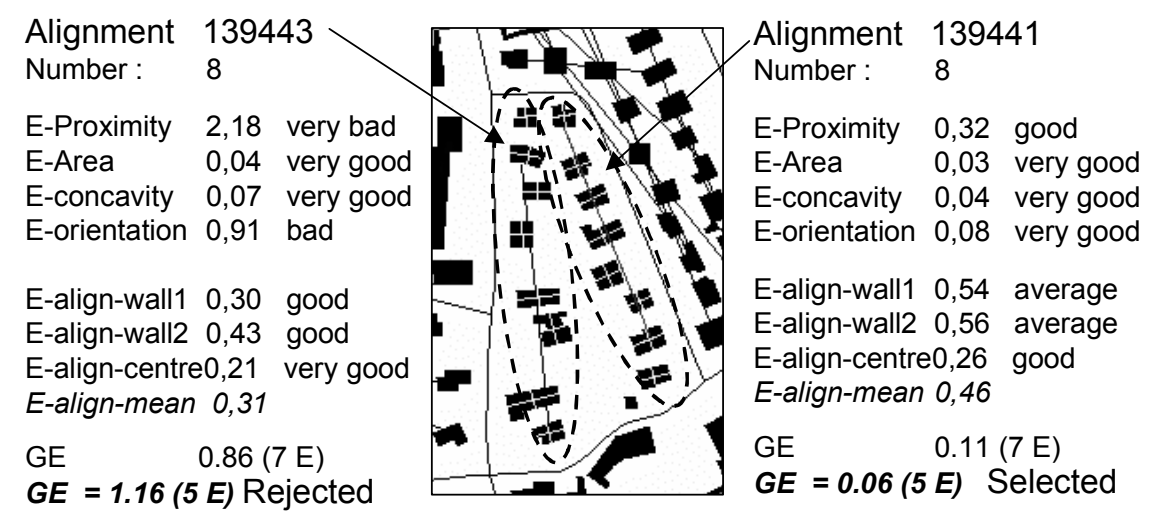

Fig. 9. Visual results : example 1 


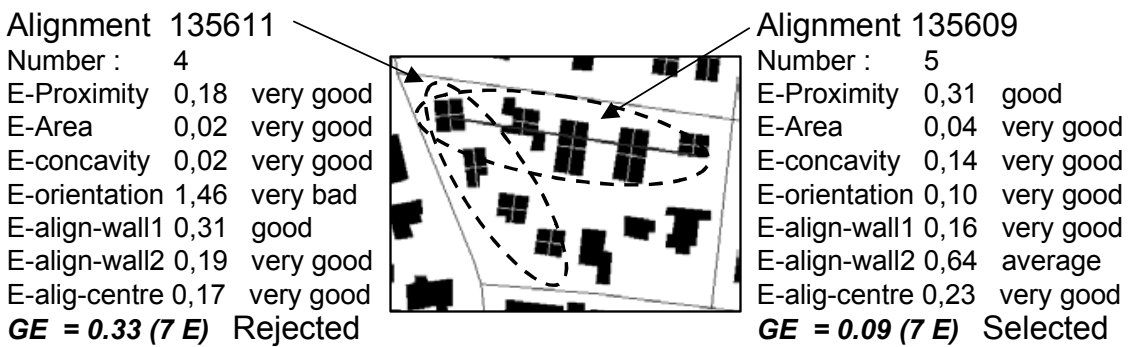

Fig. 10. Visual results example 2

Without having addressed optimisation concerns, we observe that some estimators $E_{i}$, are still inadequate. For example,

- The Area and Concavity estimator values are always too small. Thus, the normalisation should be reconsidered.

- The estimator of alignment (E-align) based on the variation of angles that connects close buildings is not as strict as a visual inspection might require, (e.g. Fig. 9, alignment 139443). Indeed the visual perception of alignment depends not only on angles but also on distances. A solution to improve E-align lies in replacing $\alpha$ by sinus $\alpha$. Moreover; a combination of the three indicators should be developed. Taking the three indicators (7E) puts too much weight on alignment, while taking the average $(5 \mathrm{E})$ may be not be the best solution.

Even if the visual results are acceptable, some more tests are needed to tune these functions. The difficulty is partly related to the fact that statistical measurements have been used for only a limited number of objects.

The comparison between the orientation of the buildings and the orientation of the alignment, as well as the comparison between the orientation of the alignment and the orientation of the closest road would be useful information to add.

The aggregation of estimators for the global qualification of the perceptual alignment also needs improvements. In the above assessment, all estimators are equally weighted and an average is computed. The only exception is the distance estimator, which combines the regularity and the value of the distance. The aggregation of two concepts (regularity and value) is certainly not optimal. An improvement might be achieved using the following rule: the smallest the object size, the smallest the acceptable distance.

The detection of alignment (section 2) could include some buildings that may decrease the quality of the global structure. Therefore, these structures might be rejected during the selection step (section 3). Whenever a large structure is removed, consideration could be given to the effect it has on decreasing the global quality. 


\section{The Use of Perceptual Alignments for Generalisation}

The aim of this research was to detect building perceptual alignments for generalisation purposes. This generalisation process has not yet been implemented but an explanation of how these structures will be used is provided below:

- For building typification, minimum size and minimum interdistance, are used to find the number for required buildings. The appropriate number of buildings should be removed (either the less regular or the redundant ones) and the final alignment should be rearranged while maintaining the global characteristics of the structure.

- In case of an excessive building density, if the building block is too thin and if there are two parallel building alignment structures (which is often the case as there are two road parallel roads), we could preserve the best one and remove the buildings of the other alignment..

- Last but not least, perceptual alignments should be useful for the building displacement process. New algorithms such as [Bader 01] perform displacements based on various strengths between objects. In case of the displacement of buildings that belong to a perceptual alignment, special strengths should be introduced to preserve the alignments as much as possible.

\section{Conclusion}

In this paper, we present research on detecting and qualifying building perceptual alignments. These types of patterns have been chosen as they are frequently found in urban areas and are necessary elements in cartographic presentation and generalisation activities, such as typification and displacement. The idea is to detect the structures and to propose new and simpler algorithms that would better maintain geographical patterns. The proposed method is a top down detection approach as it starts with predefined straight-line templates and seeks buildings that participate in these structures. The detection phase gives acceptable results. The characterisation and selection phase should still be improved (see 3.3) even if the results are already reasonable sound. The concept of estimator functions requires further work and fine-tuning. The next step is to build new generalisation algorithms that will, as a result, be easier to develop as it is anticipated that the pattern derivation will resolve a number of the problem issues.

\section{References}

Bader M (2001) Energy Minimization Methods for Feature Displacement in Map generalization. $\mathrm{PhD}$ thesis, University of Zurich, Suisse 
Boffet A, Rocca Serra S (2001) Identification of spatial structures within urban blocks for town qualification. In: Proceedings XX International Cartographic Conference, Vol 3. 6-10 August, 2001, Beijing, China, pp 1974-1983

Hangouët JF (1998) Approche et méthodes pour l'automatisation de la généralisation cartographique ; application en bord de ville. Ph.D. thesis, University of Marne-LaVallée France ${ }^{1}$

Regnauld N (1998) Généralisation du bâti: Structure spatiale de type graphe et représentation cartographique. Ph.D. thesis, University of Provence - Aix-Marseille $1^{1}$

Ruas A (1999) Modèle de généralisation de données géographiques à base de contraintes et d'autonomie. Ph.D. thesis, University of Marne-La-Vallée France ${ }^{1}$

Sester M (2000) Generalization based of least squared adjustments. In: Proceedings International Archives of Photogrammetry and Remote Sensing, 16-23 July 2000, Amsterdam, The Netherlands, 23: 931-938

Wertheimer M (1912) Über das Denken der Naturvölker: I. Zahlen und Zahlgebilde. Zeitschrift für Psychologie, 60:321-378. Reprinted in Drei Abhandlungen zur Gestalttheorie, 1925

${ }^{1}$ See ftp://ftp.ign.fr/ign/COGIT/THESES/ 